\title{
Die Messung von Indizes in der Vergleichenden Politikwissenschaft - methodologische Spitzfindigkeit oder substantielle Notwendigkeit
}

\author{
Susanne Pickel · Gert Pickel
}

Zusammenfassung: Indizes haben in den letzten Jahrzehnten in der Vergleichenden Politikwissenschaft einen regen Aufschwung genommen. Aus einigen Bereichen, wie z.B. der vergleichenden Demokratiemessung, sind sie heute nicht mehr wegzudenken. Ihre Vorteile liegen in der Verdichtung und damit der Komplexitätsreduktion von Einzelinformationen und der Vermeidung von Multikollinearität. Gleichzeitig unterliegen sie einigen Problemen. Konzeptionalisierungsund Aggregationsprobleme sind nur die auffälligsten davon. Der Artikel fasst die Überlegungen der verschiedenen Autoren des Sonderheftes der Zeitschrift für Vergleichende Politikwissenschaft zur Bedeutung von Indizes in der Vergleichenden Politikwissenschaft zusammen und bilanziert die zentralen Forderungen an eine zukünftige Verwendung. Diese lassen sich mit den Begriffen Kontrolle der Messqualität, Theorieanbindung, Konzentration der Erkenntnisorientierung, methodische und politische Reflexion verbinden.

Schlüsselwörter: Vergleichende Politikwissenschaft · Indexbildung $\cdot$ Methoden

\section{The measurement of indices in comparatice politics - methodological sophistry or substantial necessity}

\begin{abstract}
In the last decades, indices boomed in comparative politics. Comparative measurement of democracy would not work without the variety of indices that poped up from the scietific community. Condensation and reduction of complexity of single information and avoiding multicollinearity are their main advantages of indices. At the same time, they produce some problems of conceptualization and aggregation. The article collects the ideas of different authors of this special issue of the journal for comparative politics and sums up their central demands for future use of indices. The demands include quality of measurement, close links to theory, concentration on scientific conclusion as well as methodological and political reflection.
\end{abstract}

Online publiziert: 18.09 .2012

(C) VS Verlag für Sozialwissenschaften 2012

Prof. Dr. S. Pickel $(\bowtie)$

Institut für Politikwissenschaft, Universität Duisburg-Essen,

Forsthausweg 2, 47057 Duisburg, Deutschland

E-Mail: susanne.pickel@uni-due.de

Prof. Dr. G. Pickel

Institut für Praktische Theologie, Abteilung Kirchen- und Religionssoziologie, Universität Leipzig,

Otto-Schill-Str. 2, 04109 Leipzig, Deutschland

E-Mail: pickel@rz.uni-leipzig.de 
Keywords: Comparative politics $\cdot$ Index-construction $\cdot$ Methods

\section{Indizes in der Vergleichenden Politikwissenschaft - pro und kontra Datenreduktion}

In der Vergleichenden Politikwissenschaft wird in den letzten Jahrzehnten verstärkt und auf fast allen Gebieten mit Indizes und Kennziffern unterschiedlichster Art gearbeitet. Teilbereiche der Vergleichenden Politikwissenschaft, wie z.B. die Demokratiemessung (siehe unter anderen Lauth 2004; Pickel und Pickel 2006; Munck 2009; Teorell 2010), erzielen ihre Ergebnisse mit Hilfe dieser Form der Zusammenfassung und Komprimierung von Informationen. In den letzten Jahren hat sich dabei die Diskussion verstärkt sogar in Richtung einer Bewertung der Methode der Indexbildung gewendet. Statt des (gemessenen) Inhalts und der inhaltlichen Ergebnisse sind Debatten über die Reliabilität und Validität der Instrumente der Demokratiemessung in den Vordergrund getreten (s. Munck und Verkuilen 2002; Pickel und Müller 2006; Müller und Pickel 2007; Fuchs und Roller 2008).

Doch auch aus anderen Sektoren der Vergleichenden Politikwissenschaft sind Indizes nicht mehr wegzudenken. So wird die Stabilität politischer Systeme (fragility indices), die Bestimmung von Transformationserfolgen (Bertelsmann Transformation Index, BTI), die Qualität von Demokratien (Sustainable Governance Index, SGI; Democracy Barometer, DB) oder auch von Autokratien (mit teilweise Messung Hadenius und Teorell 2006, 2007; Teorell 2010) oder die Korruptionsbestimmung (Corruption Perception Index, CPI, ermittelt von Transparency International) weitgehend über Indizes durchgeführt. Die Bestimmung von Humanentwicklung erfolgt über eine Kombination von ökonomischen und sozialen Daten (Human Development Index, HDI). Gemeinhin hat man den Eindruck, dass die Zahl der Indizes exponentiell zunehmend ist.

\subsection{Vorteile}

Was sind die Gründe für diese Ausbreitung von Indizes? Vor allem ihre objektiven Vorteile gegenüber einzelnen Kennzahlen. Die Vorteile liegen dabei auf der Hand: Mehrere Einzelinformationen werden zu einer einzigen Aussage gebündelt, die komplexe Informationen reduziert und eine mehrdimensional abgesicherte Information enthält. Die Aussage des jeweiligen Index ermöglicht somit Erkenntnisse über ein Syndrom gesellschaftlicher und/oder politischer Zustände. Durch diese Informationskomprimierung ermöglichen Indizes einen stringenten und gesicherteren Vergleich über die Zeit oder zwischen Einheiten (z.B. Ländern oder Kulturen) und eine statistische Weiterbearbeitung. Wie anders wären sonst die vielen Einzelinformationen in weiterführende Analysen einzubeziehen? Die Ermittlung von strukturellen Beziehungen auf der Makroebene ist ohne Indizes fast undenkbar. Wie soll sonst bspw. der potentielle Zusammenhang zwischen Modernisierung und Demokratisierung überprüft werden oder ökonomische und kulturelle Interdependenzen für den politischen Sektor sichtbar gemacht werden?

Aus vergleichender Perspektive können somit Entwicklungen und Bündelungen der Performanz vieler Länder abgebildet werden, ohne dass sich Forscher und Nutzer in 
Einzelinformationen verzetteln müssen. Und noch ein recht praktischer Vorteil kommt in jüngerer Zeit hinzu: Die komprimierten Informationen stehen i.d.R. auf den Internetseiten der Indizes zur Verfügung, so dass auch vertiefende Länderanalysen und die Darstellung zeitlicher Entwicklungsverläufe leicht möglich sind. Und dies sogar für Studierende in Seminaren! Vermehrt kann man auch die Informationen erhalten, die in die Indizes eingehen. Oft erschließt sich erst aus dem Wechselspiel der hochaggregierten Maßzahlen und der disaggregierten Einzelinformationen das volle Potenzial der Indexanwendung. Damit werden aber auch Abweichungen kulturspezifischer oder politischer Art identifizierbar. Besitzt z. B. ein politisches Regime seine Defizite in der Gewährung von demokratischen Rechten, wie Religionsfreiheit oder Pressefreiheit, so finden sich in einem anderen Regime zwar diese Rechte, aber Korruption und Klientelismus schränken die demokratische Qualität ein.

Bei den Maßen zur Bestimmung der demokratischen Qualität politischer Systeme fällt auf, dass die verwendeten Demokratiebegriffe sich stark einander annähern. So wird z. B. die Vorstellung von der Rechtsstaatlichkeit wird inzwischen als Bestandteil der Demokratie anerkannt, zuweilen treten wirtschaftliche und soziale Performanz als ,added values“ hinzu (BTI, SGI). Selbst wenn diese Konvergenz mit einer gewissen Vorsicht zu betrachten ist, gibt sie auch Hinweise auf scheinbar existierende Überschneidungen im Verständnis bestimmter Themen und Untersuchungsinhalte. Sie dient quasi als Katalysator einer Reflexion über den Untersuchungsgegenstand. Andere Indizes wieder bündeln eine hohe Zahl an (Demokratie)Indikatoren (DB) aus vielen Quellen. Sie werden immer öfter in disaggregierter Form angeboten und erlauben es somit den Nutzern die Indikatoren eigenständig zu kombinieren. Die Vielfalt der verfügbaren Maße ermöglicht die Abbildung kontinuierlicher Übergänge zwischen den politischen Systemen (Freedom House, PolityIV, BTI), die Darstellung der Qualität ausschließlich von Demokratien der OECD-Welt (SGI, DB) oder die Kombination beider Informationen (Neuer Index Demokratie (NID)).

Indizes bündeln nicht nur Informationen, sie bieten bei korrekter Gestaltung und klarer theoretischer Herleitung der Zusammensetzungskriterien auch den methodischen Vorteil einer größeren Stabilität und geringerer Beeinflussbarkeit durch Zufallsschwankungen. Zudem kommt man um einen Index nicht herum, wenn die zu prüfende sozialwissenschaftliche These mehrere Dimensionen einbezieht oder aber ein Indikator die zugrundeliegende Fragestellung nur ungenügend abbilden kann (vgl. Schnell et al. 2005).

\subsection{Anwendungsprobleme}

Doch resultiert aus der Verfügbarkeit einer hohen Anzahl an komplexen Maßzahlen auch deren problemlose Anwendbarkeit? Der bereits erfolgte Verweis auf die Verlagerung der Debatten innerhalb der Demokratiemessung bzw. der Qualitätsbestimmung politischer Systeme lässt hier Zweifel aufkommen. Entsprechend sind hinsichtlich der Verwendung von Indizes in der Vergleichenden Politikwissenschaft einige Schwierigkeiten zu identifizieren: So erweist es sich als problematisch, dass der Nutzer der Indizes, der selten selbst an der Zusammenstellung des verwendeten Index beteiligt ist, häufig nicht genau weiß, welche Informationen in welcher Weise in die Indexkonstruktion eingeflossen ist. Teils sind die Quellen der verwendeten Indikatoren des Index nicht nachzuvollziehen, teils beruht der Index auf unsicheren Datenbeständen. Damit bleiben Erkenntnisse über 
die Validität und Reliabilität dieser Indizes sowie deren Prüfung eingeschränkt. Die Verbindung zur Ursprungsintension des Indexkonstrukteurs entschlüsselt sich eher mühsam über umfangreiche Recherchen und Plausibilitätskontrollen oder durch konkrete Kontakte zum Indexkonstrukteur. Ansonsten bleibt beim sekundäranalytischen Zugang zum verarbeiteten empirischen Material häufig die Sicht auf die Konstruktionsmechanismen - und insbesondere die eingehenden Datenressourcen - versperrt. Einfach gesagt: Man weiß nicht wirklich, mit welchen Informationen man als Forscher weiterarbeitet - und wie belastbar ein damit erzieltes Ergebnis wirklich ist (Transparenzproblem).

In solchen Fällen stellt sich zwangsläufig eine weitere Frage: Messen die Indizes wirklich das, was sie messen wollen und - aus Sicht des Forschers - auch sollen? Hier geht es um die Validität der Messung im Sinne einer möglichst genauen Abbildung dessen, was man abzubilden angibt. Messen bspw. sog. Demokratieindizes auch tatsächlich die Qualität einer Demokratie oder nicht doch eher die Freiheit der Bürger, die oft mit Demokratie einhergeht (Freedom House)? Oder wird „lediglich“ eine Institutionenkonstellation auf dem Papier einer Verfassung bestimmt (Polity IV), die zusätzlich einen Schwerpunkt auf die Einhegung der Exekutive durch institutionalisierte Kontrollmechanismen legt? Welche Merkmale der Demokratie sind die wesentlichen (NID) und welche Kriterien sind eher Folgen demokratischer Regime als ihre Kennzeichen (DB, SGI, EIU Democracy Index)? Und wo liegt ein angemessener Mittelweg zwischen allumfassender Messung (Democracy Barometer) und minimalistischer Projektion (Index of Democratization, ID)?

An dritter Stelle steht die Zuverlässigkeit (Reliabilität) der Messung, die bestimmten Richtlinien der Indexkonstruktion folgen und im Wiederholungsfall durch andere Forscher zu einem zumindest sehr ähnlichen Ergebnis führen sollte. Eine Auseinandersetzung mit diesen drei Grundkriterien der empirischen Forschung scheint von so grundsätzlicher Bedeutung, dass es doch verwundert, wie selten sie reflektiert werden. Gelegentlich findet sich in empirischen Studien an dieser Stelle des Forschungsprozesses ein teilweise fast naiver Glaube an die Gültigkeit und Zuverlässigkeit verfügbarer Indizes. Dies gilt insbesondere, wenn sie von größeren Forschungsinstitutionen stammen. Zuweilen unterscheiden sich jedoch selbst „einfachste“ Grunddaten wie das Bruttoinlandsprodukt pro Kopf in Kaufparitäten je nach der gewählten Quelle (EUROSTAT, Weltbank oder auch nationale Quellen). Leider kann sich dieses ,zu wenig“ an Reflexion in massiven inhaltlichen Fehlinterpretationen über gesellschaftliche und politische Tatbestände und Entwicklungsprozesse niederschlagen. Richtigerweise investieren viele Forscher große Mühen, um hinter das Geflecht der zuweilen extrem komplex zusammengesetzten Indizes blicken zu können. Dieses Unterfangen ist dabei weder einfach, noch immer befriedigend, führt es doch oft zu Verunsicherungen über das verwendete Instrumentarium.

\subsection{Redundanzen}

Ein weiteres Problem des derzeitigen Umgangs mit Indizes in der Vergleichenden Politikwissenschaft ist, dass verschiedene Indizes zu den gleichen Forschungsbereichen (Demokratie, Parlamentarismus) bereitstehen. Dieser Pluralismus ist an sich kein Problem. So scheint es ja im Gegenteil günstig, unterschiedliche, sich wechselseitig kontrollierende Abbildungen eines Gegenstandes zur Verfügung stehen zu haben. Die Schwierigkeit liegt vielmehr darin, dass die (aufwendigen) Konstruktionsleistungen von den Forschern nur 
begrenzt wechselseitig rezipiert werden. Teilweise stehen die Konstrukte in einem Konkurrenzverhältnis zueinander, wobei mittlerweile auch das Werben um knapper werdende Forschungsmittel eine Rolle spielen dürfte. Die Folge ist, dass verschiedene Erhebungen ein und desselben Forschungsgegenstandes teils bezugslos nebeneinander stehen. So bleibt es dem jeweiligen Nutzer weitgehend selbst überlassen, welchen Index er zur Messung (von Demokratie oder Autokratie) verwendet. Die Auswahl ergibt sich entweder aufgrund spezieller Vorlieben des Forschers oder ist einfach dem Umstand geschuldet, dass nur eine selektive Kenntnis über die verfügbaren Indizes vorliegt. Womit nicht ausgeschlossen ist, dass auch eine profunde, reflektierte und gezielte Indexauswahl erfolgen kann.

Der zuletzt genannte Zustand hat sich in den letzten Jahren, vor allem aufgrund des Internets und der angestiegenen Verfügbarkeit von Indizes, wesentlich gebessert. Der Zugriff ist einfacher geworden, die Transparenz höher. Doch immer noch gilt: Wenn man nicht auf klare Gütekriterien zurückgreifen kann, fällt die Wahl des verwendeten Index häufig der Zufälligkeit anheim - oder der leichten Verfügbarkeit von möglichst vielen Daten zu möglichst vielen Zeitpunkten. ${ }^{1}$ Die Vielfalt ist mittlerweile so groß, dass kaum eine Forschungsarbeit, z. B. in der Demokratiemessung, mit nur einem Index auskommt. Häufig werden gleichzeitig mehrere Indizes verwendet oder sie werden wieder statistisch zu einem „Metaindex“ zusammengefasst. Stellt ersteres möglicherweise ein kleineres Problem dar, denn die wechselseitige Kontrolle mit verschiedenen Instrumenten kann ein Reliabilitätskriterium darstellen, so muss man sich im zweiten Fall oft fragen, ob man mit einem „Metaindex“ nicht auch die Probleme der Einzelindizes multipliziert. Ist dies der Fall, dann kommt noch das Manko immer geringerer Sparsamkeit der Messung - ein nicht zu unterschätzendes Kriterium moderner Forschung und Indexbildung - hinzu.

\subsection{Operationalisierung und Prüfung}

Bei der Verwendung von komparativen Indizes sind auch immer wieder massive Probleme auf der Ebene der Rückbindung an die Theorie (= Inhalt) und auf der Ebene der Konstruktion (= Methodologie) festzustellen. Die Konstruktionsmechanismen sind oft uneinheitlich und die Konstrukte (Indizes) selbst beinhalten häufig erhebliche theoretisch-ideologische Vorentscheidungen. Am deutlichsten wird dies in den Debatten der (angeblich) ethnozentristischen Messindizes zur Demokratiequalität, aber auch bei der politischen Relevanz von fragility indizes für die Entwicklungshilfe oder sozioökonomischen Indizes für die Zuweisung von finanziellen Mitteln durch internationale Organisationen. Gelegentlich verschwimmt auch der Bezug zwischen dem konstruierten Index und der dahinterstehenden Theorie. Oder methodologisch gesprochen, das zu messende latente Konstrukt und die Fragestellung decken sich nicht. Nicht, dass solche Probleme ungesehen bleiben. Prüfungen und Tests sind in der mit Indizes arbeitenden Vergleichenden Politikwissenschaft mittlerweile weit verbreitet. Aber oft wird immer noch zur Stützung der (auch selbst konstruierten) Indizes (fälschlicherweise) die Reliabilität als Ersatz der Validität herangezogen. Dies führt uns ziemlich direkt zum Ziel des vorliegenden Sonderbandes. Wir möchten dazu auffordern, sich

1 Die starke Ausbreitung der Messung von Freedom House für die Messung der Demokratiequalität ist ein gutes Beispiel hierfür. 
a. der Problematiken und Risiken, aber auch des Nutzens von Indizes in der Vergleichenden Politikwissenschaft gewahr zu werden (methodologische Seite) ${ }^{2}$ sich

b. über Indizes zu informieren, die im Bereich der Vergleichenden Politikwissenschaft aktuell verwendet werden und diese hinsichtlich ihres inhaltlichen Nutzens zu diskutieren (inhaltliche Seite),

c. der konkreten methodischen Konstruktion dieser Indizes zu vergewissern, darüber zu diskutieren und ggf. zu Verbesserungen beizutragen (methodische Seite),

d. über Konstruktionsprinzipien sowie theoretische Rückbindung von Indizes auszutauschen, da nicht wenige auf den gleichen Gebieten vorliegen (Austausch- und Diskussionsseite).

Diese Anliegen werden von einem einfachen Ziel begleitet. So hat sich die Zahl der Indizes so vermehrt, dass dem interessierten Forscher oft die Übersicht verlorengeht. Auch hier soll der vorliegende Band, zumindest in den ihm möglichen Grenzen, Abhilfe schaffen.

\section{Wo findet man Indizes?}

Spricht man von Indizes, so ist der Begriff des „Messens“ stets implizit. Unter Messen wird dabei die Zuordnung von Zahlen zu Objekten (Schnell et al. 2005, S. 138-139) verstanden. Davon zu unterscheiden sind Kategorien- und Typenbildungen. Kategorien sind Oberbegriffe, denen bestimmte Variablen zugeordnet werden. So können unterschiedliche Regierungssysteme unter die Oberbegriffe „Demokratie“ und „Autokratie“ gefasst werden. Kategorien können selbst wiederum als Variablen Verwendung finden. Sie werden dabei durch einfache oder komplexe Oberbegriffe gebildet. Eine spezifische Form von Kategorien bilden Typen und Typologien. Unter einer Typologie wird die Zuordnung von mindestens zwei Merkmalen, die zwei oder mehr Ausprägungen aufweisen, zu einer spezifischen Kombination der einzelnen Merkmalsausprägungen verstanden (Aarebrod et al. 1997, S. 62; Nohlen 1994, S. 491-492). ${ }^{3}$ Ein Merkmal bzw. seine Ausprägung kann dabei direkt beobachtbar sein (z. B. Anzahl der Herrschenden) oder als latente Variable vorliegen und benötigt dann eine weitere Operationalisierung mit entsprechenden Indikatoren. (Lauth et al. 2009, S. 44). Solche Typologisierungen finden häufig in der Autokratieforschung Verwendung. Autokratien werden dann nicht nach dem Grad der Geschlossenheit des politischen Systems, sondern nach der Zusammensetzung von Systemeigenschaften (Merkmalen) unterschieden. Beispiele aus der aktuellen Forschung sind das DemocracyDictatorship-Projekt (Cheibub et al. 2009, aufbauend auf Przeworski et al. 2000), die Typologie autoritärer Regime nach Geddes (1999) (Geddes et al. 2011), die Typologie von Hadenius und Teorell $(2006,2007)$ und die Typologie von Kailitz (2009a, b, 2012).

2 Dabei beziehen sich die methodischen Anmerkungen häufig nicht allein auf die vergleichende Politikwissenschaft. Vielmehr besitzen sie Bedeutung für den Umgang mit Indizes überhaupt. Diese Diskussion soll hier allerdings nicht geführt werden und eine Konzentration auf den Indizes der vergleichenden Politikwissenschaft liegen.

3 Liegt nur ein Merkmal mit verschiedenen Ausprägungen vor, dann wird von einer Klassifikation gesprochen. 
Abb. 1: Beispiele für Indizes

\begin{tabular}{|l|}
\hline Bertelsmann Transformation Index (www.bti-project.de/) \\
\hline Corruption Perception Index (CPI; cpi.transparency.org/cpi2011/) \\
\hline Democracy Barometer (BTI; www.democracybarometer.org/) \\
\hline The Economist Intelligence Unit's Index of Democracy \\
\hline (graphics.eiu.com/PDF/Democracy_Index_2010_web.pdf) \\
\hline Failed States Index (Index Fragile Staatlichkeit; www.fundforpeace.org/global/) \\
\hline Freedom House (www.freedomhouse.org/) \\
\hline Human Development Index (HDI; hdr.undp.org/en/humandev/) \\
\hline Index of Democratization \\
\hline (www.prio.no/CSCW/Datasets/Governance/Vanhanens-index-of-democracy/) \\
\hline Polity IV (www.systemicpeace.org/polity/polity4.htm) \\
\hline Sustainable Governance Indicators (SGI; www.sgi-network.org/) \\
\hline Worldwide Governance Indicators \\
\hline (WGI; info.worldbank.org/governance/wgi/index.asp) \\
\hline
\end{tabular}

In welchen inhaltlichen Bereichen der Vergleichenden Politikwissenschaft finden Qualitätsmaße Verwendung? Ein Kernbereich der Index- bzw. Typologiennutzung liegt in der Bestimmung der Erfolge von Policies: Hier sind nur exemplarisch die Arbeiten von Francis Castles (1998) zu nennen, der policy-outcomes in Abhängigkeit einer Vielzahl von ökonomischen, sozialstrukturellen und kulturellen Variablen ausmacht. Doch auch im Bereich der Polity kommen Indizes zur Anwendung. Messungen der Fragilität von Staatlichkeit sind genauso gebräuchlich, wie die Abgrenzung politischer Systeme. Herausragendes Beispiel hierfür sind Messinstrumente von Polity IV. Daneben sind die Indizes der Weltbank zu beachten, die sich in den Worldwide Governance Indicators manifestieren (siehe hierzu ausführlich den Beitrag von Muno in diesem Band).

Damit nähert man sich sicherlich dem derzeitigen Zentralbereich der Verwendung von Indizes in der Politikwissenschaft an - der Demokratiemessung. Dort hat sich eine Vielzahl unterschiedlicher Vorgehen etabliert. Nicht von ungefähr konzentrierte sich die Debatte über die Kriterien von Indexmessung stark auf die Demokratiemessung (Munck und Verkuilen 2002; Müller und Pickel 2007). Das bedeutet nicht, dass hier die Anwendungsbreite endet. So werden sowohl das Verhältnis von Kirche und Staat (s. Traunmüller in diesem Band), die Abbildungen von Aspekten der politischen Kultur und der Einstellungsforschung wie auch Rahmenbedingungen struktureller und kultureller Ausprägung mit Indizes erfasst. Und nicht zu vergessen sind die auf dem ökonomischen Sektor vorgenommenen Konstruktionen.

\section{Was ist bei der Indexkonstruktion und ihrer Überprüfung zu beachten?}

Der Umgang mit Indizes sowie die Beurteilung ihrer Qualität rückte seit dem Beitrag von Munck und Verkuilen 2002 verstärkt ins Blickfeld auch der deutschen Vergleichenden 
Politikwissenschaft. Die Artikel von Müller und Pickel (2007), Pickel und Müller (2006) und Fuchs und Roller (2008) griffen diese Problematisierung auf. Die Ergebnisse sind eindeutig: Trotz unterschiedlicher Schwerpunkte wird versucht, ein „Prüfset“ an Kriterien festzulegen, nach denen (ursprünglich) Demokratieindizes in ihrer Qualität bewertet werden können. Will man die im Folgenden vorgestellten Indizes beurteilen, so bietet es sich an, diese Kriterien genauer zu beachten (Pickel und Müller 2006 mit Ergänzungen). Sie sind prinzipiell zur Evaluierung aller wissenschaftlichen Indizes geeignet.

1. Phase der Konzeptionalisierung (Sparsamkeit, Relevanz, Redundanz und Zuordnung beziehen sich auf die Konzeptspezifikation und Konzeptlogik)

\begin{tabular}{|c|c|c|}
\hline $\begin{array}{l}\text { Identifikation der Demokratie- } \\
\text { Merkmale (1. System- } \\
\text { Definition; } 2 \text {. Merkmale } \\
\text { und ihre Komponenten) }\end{array}$ & $\begin{array}{l}\text { Konzeptspezifikation: Ver- } \\
\text { meidung minimalistischer oder } \\
\text { maximalistischer System- } \\
\text { Definitionen (Sparsamkeit, } \\
\text { aber alle im Hinblick auf das } \\
\text { theoretische Konzept } \\
\text {,relevanten' Merkmale) }\end{array}$ & $\begin{array}{l}\text { Sparsamkeit } \\
\text { Relevanz }\end{array}$ \\
\hline $\begin{array}{l}\text { Horizontal und vertikal logische } \\
\text { Organisation der Merkmalskom- } \\
\text { ponenten nach Abstraktionsgrad } \\
\text { und Merkmalszugehörigkeit } \\
\text { („Konzeptbaum“) }\end{array}$ & $\begin{array}{l}\text { Konzeptlogik: Vermeidung von Redundanz } \\
\text { (Dopplungseffekten) und falscher } \\
\text { Zuordnung der Merkmalskomponenten }\end{array}$ & $\begin{array}{l}\text { Redundanz } \\
\text { Zuordnung }\end{array}$ \\
\hline
\end{tabular}

Sparsamkeit ist v.a. dann gegeben, wenn sich ein Konzept auf das Wesentliche eines politischen Systems beschränkt. Weitere Elemente wie z. B. sozio-ökonomische Faktoren stellen bei vielen kausalen Untersuchungen abhängige oder unabhängige Variablen dar, die folglich nicht als Gegenstand des Index bzw. des politischen Systems verwertet werden sollten. Relevanz bezieht sich auf die Grunddefinition der gemessenen politischen Systeme. Indikatoren, die nicht Bestandteil dieser Definitionen sind, gehören auch nicht in den Index zur Systembestimmung.

Merkmale sollten logisch entlang eines Konzeptbaumes organisiert sein. Merkmale gleichen Abstraktionsgrades gehören auf eine Messebene; sie sollten zudem nach theoretischer Zugehörigkeit zu Oberbegriffen zugeordnet werden (Zuordnung). Das Konzept sollte in trennscharfe Dimensionen ausdifferenziert sein. Dabei gilt es, Dopplungen von Merkmalen bzw. Mehrfachmessungen einzelner Komponenten zu vermieden (Redundanz). 
2. Phase der Messung (Indikatorenvalidität, Messniveau, Dokumentation, Replizierbarkeit und theoretische Begründung beeinflussen die Qualität der Messung)

\begin{tabular}{|c|c|}
\hline \multirow[t]{2}{*}{$\begin{array}{l}\text { Auswahl der Indikatoren } \\
\text { (Umwandlung der Merkmals- } \\
\text { komponenten bzw. latenten } \\
\text { Variablen zu beobachtbaren } \\
\text { Variablen bzw. Indikatoren = } \\
\text { Operationalisierung) }\end{array}$} & $\begin{array}{l}\text { Validität: Verwendung multip- } \\
\text { ler (polyvalenter) Indikatoren } \\
\text { mit kulturellen Äquivalenten } \\
\text { (Spannung zw. kulturellem } \\
\text { Kontext und Vergleichbarkeit); } \\
\text { Auswahl von Indikatoren } \\
\text { unter Berücksichtigung des } \\
\text { Quellenproblems (Beachte: } \\
\text { Bias durch historisch kon- } \\
\text { tingente und kontextrelative } \\
\text { Überlieferung! Möglicher Bias } \\
\text { durch Blickwinkel aus „Sicht“ } \\
\text { der Demokratie) }\end{array}$ \\
\hline & Reliabilität \\
\hline
\end{tabular}

Auswahl des Messniveaus (Skalierung) der Kodierung

Validität: Maximierung von Homogenität innerhalb der Messklassen (Vermeidung zu fein- oder grobkörniger Skalierungen, je nach Datenlage, aber so, dass verschiedene Fälle auch als solche sichtbar bleiben)

Dokumentation des Kodiervorganges (v. a. der Kodierregeln und disaggregierten Daten)
Reliabilität/Replizierbarkeit:

Vermeidung von systematischen Messfehlern, die die Validität beeinträchtigen Möglichst direkter Verweis auf Internetzugang der Quelle Bei mehreren möglichen Quellen: Warum wurde eine bestimmte Quelle gewählt?

Mehrere Kodierer bestimmen den Messwert unabhängig voneinander

Leichte Verfügbarkeit disaggregierter Daten

\author{
Messen die Indikatoren \\ richtig? \\ Breite der Quellenbasis
}

Theoretische Begründung des
Messniveaus

Kodierregeln

Quellenangabe

Auswahl der Quellen

Reliabilitätstests zw. Kodierern

Disaggregierte Daten

Bei der Auswahl der Indikatoren ist auf die Erfassung aller Merkmale des Konzeptes zu achten. Die Indikatoren sind zunächst aus dem Konzept abzuleiten und ihre Bestimmung (Messung oder Expertenbewertung) zu erläutern. Die Indikatoren sollten die konzeptbestandteile, denen sie zugeordnet sind, auch tatsächlich messen (Validität), sie sollten als Indikatoren anwendbar und trennscharf gestaltet sein.

Die Daten sind auf breiter Basis auszuwählen, Quellen staatlicher und nicht-staatlicher Autoren sollten ebenso gemischt werden wie Quellen mit unterschiedlichem nationalem und kulturellem Hintergrund. Das Beispiel Syrien zeigt aktuell, wie wesentlich diese For- 
derung an einen reliablen, validen und transparenten Index ist. Einseitige Quellenauswahl führt zu einseitigen Indizes, die der Realität nicht gerecht werden.

Das Messniveau ist theoretisch zu begründen: Weshalb wird eine Klassifizierung oder eine diskrete Messung angestrebt? Oder werden Typen eines bestimmten Systems unterschieden, d.h. mit nominalem Messniveau gearbeitet? Wird die jeweilige Art der Qualitätsbestimmung dem theoretischen Konzept gerecht? Welche Skalierung ist den gewählten Indikatoren zueigen?

Um diese Auswahl bzw. die Skalierung zu belegen, müssen die Kodierregeln angegeben und trennscharf gestaltet werden. Insbesondere die Zuordnung von Zahlenwerten zu qualitativen Einschätzungen ist eindeutig zu belegen. Quellen sind anzugeben, eine Auswahl aus mehreren möglichen Quellen ist zu begründen. Reliabilitätstests sollten zwischen mehreren Kodieren durchgeführt werden, die möglichst gleich oder sehr ähnliche Ergebnisse erzielen sollten. Das Angleichen von Abweichungen ist zu begründen und zu belegen. Die Daten aller Indikatoren sind zu veröffentlichen und den Nutzern der Indizes möglichst im Internet zugänglich zu machen.

3. Phase der Aggregation (Aggregationsniveau und -regeln, Dokumentation und Nachvollziehbarkeit steigern den Erkenntiswert des Index)

Auswahl des

Aggregationsniveaus

Auswahl der

Aggregationsregel

Dokumentation der Aggregationsregeln und des Aggregationsvorganges

Stufenweise Verrechnung der Indikatoren

Verwendung unterschiedlicher Daten
Validität: Balance zwischen
Vergleichbarkeit (hohes Ag-
gregationsniveau; ,sparsamer ${ }^{6}$
Index) und möglichst genauer
Abbildung der Realität/
Varianz (niedriges Aggrega-
tionsniveau); hohes Niveau =
Informationsverlust

Validität: Beachtung der
Korrespondenz zwischen
theoretischem Zusammenhang
der System-Merkmale (In-
dikatoren) und Aggregations-
regel (soll Zusammenhänge
widerspiegeln)
Reliabilität/Replizierbarkeit

Reliabilität/Replizierbarkeit

Aggregationsregel und

Anwendbarkeit

Theoretische Begründung der

Aggregationsregel

Reliabilität/Replizierbarkeit

Subscores

Reliabilität/Replizierbarkeit

Kombination verschiedener Datenarten

Bei der Wahl des Aggregationsniveaus ist auf die Angemessenheit zu achten; die Vergleichbarkeit der Indexwerte zwischen den Ländern ist ebenso zu gewährleisten wie die Abbildungsgenauigkeit der Messung. Bei der Aggregationsregel ist, auch unabhängig von ihrer Angemessenheit, auf eine Begründung zu achten, die aus dem theoretischen 
Grundkonzept des Index hergeleitet wird. Die Aggregationsregel muss erläutert werden, so dass Vergleichsmessungen des Index möglich sind. Dies gilt auch, wenn Subscores gebildet werden. Werden verschiedene Datenarten kombiniert, so ist auch hier auf die jeweilige Skalierung, ein angemessenes Aggregationsniveau und die verwendeten Aggregationsregeln zu achten.

4. Praxis - Anwendbarkeit des Index

\begin{tabular}{lll}
\hline Anwendbarkeit & Angegeben und erreichbar & Subscores \\
Vergleichbarkeit der & Konsistente Messung im Zeitver- & Konsistenz der Fragestellung \\
Länder und über Zeit & $\begin{array}{l}\text { lauf oder rückwirkende Adaptionen } \\
\text { der Messung, wenn eine Auswei- } \\
\text { tung/Veränderung der Messung } \\
\text { erfolgt }\end{array}$ \\
\hline
\end{tabular}

Es folgt der Praxistest: Ist der Index empirisch umsetzbar? Ist der Index praktikabel d. h. nachvollziehbar und wurde er vollständig mit Subscores publiziert? Ist eine Vergleichbarkeit der Indexwerte über die gemessenen/bewerteten Länder und im Zeitverlauf möglich? Oder wurden Fragestellung und/oder Kodierung der Indikatoren verändert?

Am Ende sollte dann noch ein Bezug zum anfangs formulierten Analyseziel bestehen und die formulierten theoretischen Vorgaben eingehalten werden.

\section{Zum Buch und den Beiträgen}

Der vorliegende Sonderband beinhaltet verschiedene Beiträge, die sich in breiter Weise mit der Thematik von Indizes in der Vergleichenden Politikwissenschaft auseinandersetzen. Dabei wurde bewusst auf Beiträge verzichtet, die keinen vergleichenden Fokus aufwiesen. Zugleich sollte eine Verengung auf eine Diskussion allein innerhalb der Demokratiemessung vermieden werden Erfreulicherweise öffnete ein Call for Papers die Tür für eine doch bemerkenswerte Bandbreite an Beiträgen, die ganz unterschiedliche Möglichkeiten und Themengebiete in der Vergleichenden Politikwissenschaft aufzeigen, in denen mit Indizes gearbeitet wird. Viele der dabei zu Tage tretenden Probleme wie auch Vorteile überschnitten sich trotz dieser unterschiedlichen inhaltlichen Interessen und Zugänge.

Alle in diesem Sonderheft aufgenommenen Beiträge wurden in einer Autorenkonferenz in Leipzig diskutiert. Dann erfolgte unter Berücksichtigung der dortigen Kommentare eine Überarbeitung und Einreichung der Beiträge. In der Folge wurden die eingegangenen Beiträge in einem double-blind-peer-review-Verfahren geprüft. Allen dort mitwirkenden Gutachtern sei an dieser Stelle für ihre weiterführenden und konstruktiven Kommentare herzlich gedankt. Wie es im peer-review-Verfahren üblich ist, haben nicht alle eingereichten Beiträge den Weg in dieses Sonderheft gefunden. Dies war an einigen Stellen aufgrund der interessanten Thematiken bedauerlich. Trotzdem denken wir aber, dass diese Vorgehensweise die Richtige ist, um eine hohe Qualität der Beiträge zu gewährleisten, wie sie sich die Zeitschrift für Vergleichende Politikwissenschaft zum Maßstab gesetzt hat. Ziel des Sonderheftes war es auch, eine hinreichende Vielfalt 
und Originalität der Beiträge zu gewährleisten. Dies ist durch das geschilderte Vorgehen nicht immer gesichert. In diesem Fall scheint es gut gelungen zu sein. Fragility Indizes, ein Index zur Hochschulpolitik, Indizes zur Messung des Verhältnisses zwischen Kirche und Staat sind nur Beispiele, für die Vielfalt der Indexmessung in der Vergleichenden Politikwissenschaft. Nichtsdestoweniger stehen sie auch repräsentativ für die Präsenz bestimmter Themenbereiche. Kommen wir zu einem kurzen Überblick der in diesem Band versammelten Beiträge.

Fast alle zentralen Fragestellungen der Vergleichenden Politikwissenschaft beruhen auf der Grundannahme, dass man es bei den Untersuchungsobjekten mit Staaten zu tun hat. Diese politischen Gebilde dienen als Grundeinheit weitergehender Analysen. Dabei können diese Gebilde über ganz unterschiedliche Ausmaße an Stabilität verfügen. Dieser Frage widmet sich i.d.R. die politische Kulturforschung (zusammenfassend Pickel und Pickel 2006). Allerdings gibt sie über die Analyse von Umfragedaten zumeist nur Auskunft über eine Abschätzung der zukünftigen Stabilität. Abgesehen davon, dass auf diese Weise oft ein hoher Nutzen hinsichtlich von Aussagen über die Stabilität politischer Systeme erzielt werden kann, bleibt doch die Frage nach dem Grad der Fragilität des untersuchten Gebildes offen. Dieser Frage widmen sich in der neueren Forschung Fragilitätsindizes. Zu dieser Thematik finden sich gleich zwei Beiträge im vorliegenden Band.

Felix Bethke kommt in seiner vergleichenden Analyse der Fragilitätsindizes zu dem Befund, dass sie zwar recht zuverlässig ein empirisches Phänomen abbilden, dabei aber nicht immer klar ist, was sie genau messen. Bei näherem Hinsehen findet Bethke ein bias zugunsten eines demokratischen Verständnisses des Staates, das die Aussagekraft der meisten Indizes hinsichtlich potentieller Verfallsprozesse einschränkt. Insbesondere Aspekte der Konstruktvalidität und der theoretischen Rückbindung weisen in den meisten verfügbaren Fragilitätsmessungen Defizite auf. Dies ist umso problematischer, als dass die Transparenzprobleme einiger Ansätze die Identifikation dieser Probleme für Sekundärforscher verschleiern. Vor allem die Tendenz einiger Ansätze ,,von allem ein bisschen zu messen und den Stempel fragile Staatlichkeit draufzusetzen“ erweist sich als misslich. Ein belastbares Maß für die Bestimmung von Fragilität muss wohl - so Bethke - erst noch gefunden werden.

$\mathrm{Zu}$ einem ähnlichen Ergebnis kommt Sebastian Ziaja in seiner Beurteilung der Fragilitätsindizes. Er findet eine Gruppe ,holistischer“ Indizes, die zunächst ein gleiches Phänomen messen. Ihr empirischer Nutzen für die Erforschung von staatlicher Fragilität ist aber überschaubar, da nicht genau zu bestimmen ist, was sie messen. Dies resultiert vor allem aus dem Fehlen einer brauchbaren Definition von Fragilität. So neigen viele Messungen dazu, über maximale Konzeptspezifikationen ein „Sammelsurium“ an Indikatoren zu integrieren und dabei das Prinzip der Sparsamkeit außer Acht zu lassen. Alternative Messungen dagegen leiden daran, dass sie teilweise deutlich unterschiedliche Ergebnisse erzielen. Potentielle Weiterentwicklungsmöglichkeiten wären, einerseits den Blick stärker auf die Messung von Teilregimen zu lenken, andererseits Beziehungsanalysen zu verwandten Konzepten (politische Stabilität, Staatskapazität) vorzunehmen. Beide Autoren kommen damit trotz unabhängigen Vorgehen und anderen Zielsetzungen zu einem vergleichbaren Ergebnis.

Auch im Beitrag von Andreas Etling und Karsten Mause steht der Staat im Mittelpunkt. Ihnen geht es aber weniger um die Fragilität des Staates als um die Umsetzung 
seiner regulatorischen Kraft. Im Zentrum der kritischen Analyse stehen die in den letzten Jahren verstärkt aufkommenden Messinstrumente für das Ausmaß an regulatorischen Eingriffen in die Wirtschaft. Unterschiede bestehen insbesondere in dem verwendeten Begriff von Regulierung sowie der variierenden empirischen Reichweite der Indizes. Dabei wird auf einen Punkt hingewiesen, der für viele Indexmessungen zentral ist - die Angemessenheit des verwendeten Index für eine spezifische Fragestellung. Gleichzeitig wird deutlich, dass hier ein bedeutsamer Datenpool entsteht, der für die vergleichende Forschung erhebliche Potentiale bereithält. Gerade politikwissenschaftlich müsste dabei der Fokus noch stärker auch auf die Frage, wer reguliert, ausgerichtet werden.

Beschäftigt sich der Beitrag von Etling und Mause mit der Vermessung des regulatorischen Staates, so nimmt sich Wolfgang Muno nichts weniger vor als die Vermessung der Welt. Muno richtet seinen Blick auf die World Governance Indicators (WGI) der Weltbank. Aussagen über die Qualität der Regierungsführung oder des good governance zu erhalten, ist das Ziel dieser Indizes. Ohne Frage wird so ein breit verwendbares Tool an Daten für die komparative Analyse bereitgestellt. Allerdings ist dies nicht unproblematisch. So leiden die WGI unter einem Theoriedefizit, welches sich auf ihre Konzeptionalisierung auswirkt. Die Definition von good governance geschieht eher ad-hoc. Die Auswirkungen sind aber beachtlich, werden doch viele Entscheidungen von Organisationen, die die WGI als Entscheidungsgrundlage verwenden, auf so ebenfalls ad hoc erzielte Ergebnisse gestützt. Muno sieht hier ein maßgebliches moralisches Problem, welches in der Indexkonstruktion nicht mit bedacht wird. Das Plädoyer ist dann auch, die bestehenden Indizes nicht $\mathrm{zu}$ verwerfen, sondern zu verbessern und sich auch über ihre Verwendung bei öffentlichen Entscheidungen Gedanken zu machen.

Ein ganzes Autorenkollektiv setzt sich mit einem der Hauptprobleme der auf Indizes setzenden Vergleichenden Politikwissenschaft auseinander - der Demokratiemessung. Marc Bühlmann, Wolfgang Merkel, Lisa Müller, Heiko Giebler und Bernhard Weßels, stellen dabei ein eigenes Messinstrument vor - das Demokratiebarometer. Es soll die bestehenden Defizite und Probleme bislang verfügbarer Demokratiemessindizes zu beheben helfen. Durch eine sehr differenzierte Messung (100 Indikatoren) wird versucht, die Qualitätsunterschiede zwischen etablierten Demokratien herauszuarbeiten. Dabei wird großer Wert auf eine Anbindung an ein Theoriekonzept gelegt, welches nicht auf ein minimalistisches Verständnis von Demokratie reduziert wird. Interessant ist als Ergebnis, dass von einer Krise der Demokratie wohl kaum gesprochen werden kann, aber auch noch Entwicklungspotentiale bestehen. Und letzteres gilt nicht nur für defekte Demokratien, hybride Regime oder Autokratien.

Im Spektrum der Demokratiemessung bleiben auch Oliver Dlabac und Hans-Peter Schaub. Auch sie streben einen neuen Zugang zur Messung von Demokratie an, wollen dabei aber nicht nur - wie in der Demokratiemessung oftmals üblich - liberale, sondern auch radikaldemokratische Aspekte in Demokratien identifizieren und abbilden. Dazu konstruieren sie zwei Idealtypen von Demokratie (liberal und radikal), an deren Dimensionen die Ausrichtung (und dann auch Demokratiequalität) verschiedener politischer Systeme zu bemessen ist. Dies geschieht anhand von Netzdiagrammen. Diese ermöglichen einen dualen, mehrdimensionalen Zugang zur Frage der Messung von Demokratie. Dabei wird den Messkonzepten zur Qualität der Gedanke alternativer Formen von Demokratie zur Seite gestellt - und der oft geforderte Blick auf die theoretische Verortung gelenkt. 
Stefan Ewert betritt mit seinem Aufsatz ein völlig neues Gebiet der Indexforschung. Er untersucht anhand der Konstruktion eines Index der regionalen Vernetzung die regionale Kooperation der Hochschulpolitik im baltischen Raum. Neben dem inhaltlichen Interesse ist sein zentrales Argument für die Konstruktion eines solchen Index der Gewinn für die vergleichende Analyse gegenüber den bislang dominierenden, eher deskriptiven Regionalstudien. Deren Schwächen gerade in kausalanalytischer Hinsicht könnten auf diese Weise, wenn nicht überwunden, so doch immerhin reduziert werden. Dies hat seinen speziellen Nutzen gerade in den für die Regionalisierung bedeutsamen Diffusionsprozessen. Dies zeigen dann auch die Ergebnisse der Anwendung des Index: So kann eine Wirkung der Vernetzung der Bildungsinstitutionen genauso identifiziert werden wie allgemeinere Diffusionsprozesse. Gerade dieses Beispiel macht auch die Nützlichkeit der Verwendung von Indizes deutlich, die sich aus der Sparsamkeit und der Komprimierung der Information ergeben. So können in weiterführenden statistischen Analysen die Wirkungen verschiedener Faktoren gegeneinander getestet werden, was unter Einbezug der vielen verschiedenen Einzelfaktoren oder aufgrund der geringen Fallzahl in Regionalstudien nur sehr schwer möglich ist.

Das Verhältnis zwischen Religion und Politik, oder Kirche und Staat, zählt zu den lange etablierten Forschungsgebieten, die in verschiedenen Fächern (Politikwissenschaft, Religionssoziologie, Rechtswissenschaft) Aufmerksamkeit auf sich ziehen. Richard Traunmüller möchte durch eine Evaluation der verschiedenen Indizes, die mittlerweile die Staat-Kirche-Verflechtungen messen wollen, einen weiterführenden Beitrag leisten. Zwar sind entsprechende Messinstrumente in den letzten Jahren verstärkt aufgetaucht, doch anders als z.B. in der Demokratiemessung erfolgte eine nur begrenzte Methodenreflexion. Diese wäre aber notwendig, lassen sich doch anhand von Kernkriterien für die Indexmessung deutliche Qualitätsgrade in der Messung der Staat-Kirche-Verflechtung ausmachen. Bemerkenswert ist, dass anders als bei vielen anderen Messungen in der Vergleichenden Politikwissenschaft, bei der Staat-Kirche-Verflechtungsmessung die Hauptprobleme weniger in der Konzeptionalisierung liegen als eher in der Phase der Messung und Aggregation. Hier scheint noch ein Ausbaubedarf zu bestehen, der sich aber vor dem Hintergrund religionssoziologischer und politikwissenschaftlicher Debatten (religiöses Marktmodell) lohnen dürfte.

Jan-Erik Skanning und Jorgen Möller setzen sich im letzten Beitrag des Bandes mit der Messung von Demokratien und Nichtdemokratien auseinander. Dabei stellen sie eine Inkonsistenz zwischen Konzept und Messung fest, die in den Aggregationsregeln der Konzepte fest werden kann. Anhand der Replikation verschiedener Analysen kommen Skanning und Möller auf empirische Unterschiede, welche den Schluss nahelegen, dass hier noch einiges an Verbesserungsbedarf besteht. Sie weisen auf drei Gründe für die bestehenden Probleme und Ungenauigkeiten hin: Erstens eine teilweise fehlende methodologische Reflexion des eigenen Vorgehens und der Verwendung von extern produzierten Indizes, zweitens die Unkenntnis über die disaggregierten Werte der Indizes, drittens die oft seitens Sozialwissenschaftlern praktizierte verwirrende Verwendung von (nicht immer passenden) technischen Fachtermini. 


\section{Fazit - Indizes als nützliches aber bewusst zu gebrauchendes Instrument der Vergleichende Politikwissenschaft}

Fasst man die Ergebnisse der verschiedenen Beiträge zusammen und berücksichtigt auch die über die Beiträge hinaus vorliegenden Debatten, so werden verschiedene Punkte deutlich:

1. Indizes unterscheiden sich in ihrer Messqualität. Es gibt in der Tat bessere und auch schlechtere Indizes. Dies muss immer in Bezug auf den zu erfassenden Forschungsgegenstand beurteilt werden. Nichtsdestoweniger ist die Qualität der Messung mittlerweile nicht mehr einfach hinzunehmen und durch den Verweis auf die andere Ausrichtung des eigenen Instrumentes zu entschuldigen. Es finden sich relativ harte Kriterien, an denen man - auch reflektiert - Qualitätsgrade bemessen kann (s. Pickel und Pickel; Bühlmann et al.; Traunmüller).

2. Gleichzeitig gibt es aber auch der Forschungsfrage angemessene und weniger angemessene Indizes. Hier sind es die theoretischen wie konzeptionellen Forschungsinteressen, welche den Wert (und die Qualität) des Index vorgeben (s. Bethke; Ziaja; Schaub und Dlabac). Bedeutsam sind hier aber Kriterien der Angemessenheit für die Forschungsfrage und der Transparenz dessen, was man tut und was man verwendet.

3. Qualitätseinbußen können auf allen Ebenen der Indexkonstruktion auftreten (Munck und Verkuilen 2002; Müller und Pickel 2007). Aggregierung (s. Skanning/Möller); Konzeptionalisierung (siehe Bethke; Ziaja; Muno), Messung (s. Traunmüller) aber insbesondere der mangelnde Bezug zur Theorie und ein Root-Konzept führen zu Mängeln in der Messung. Gerade die Verzahnung zwischen Theorie und empirischer Messung sowie der Bereich der Konzeptionalisierung bedürfen in den meisten Gebieten noch einer größeren Aufmerksamkeit. So reicht es nicht aus, zuverlässig etwas zu messen, von dem man nicht weiß, ob es das ist, was man messen will (Validität).

4. Indizes sind zudem kein rein methodisches Problem. Ihre Konstruktion besitzt massive Rückwirkungen in den Forschungsprozess hinein und auf die Interpretation von Forschungsergebnissen. Politische Handlungen hängen z. T. von der Konstruktion und der inhaltlichen Zusammensetzung eines Index ab. Damit besitzen sie manifeste politische Konsequenzen - und können moralische Probleme aufwerfen (s. Muno; Etling und Mause). Indizes wie der Freedom House Index erlangen eine politische Bedeutung und können z. B. für die Vergabe von Entwicklungshilfegeldern relevant werden. Weisen die Indizes dann methodische oder konzeptionelle Schwächen auf, dann kann dies weitreichende Folgen mit sich bringen. Entsprechend muss gerade auch an die Verwertungspraxis von Indizes gedacht werden und ihre Ergebnisse sollten mit einer hinreichenden Reflexion über ihre Gültigkeit und ihre Grenzen präsentiert werden.

5. Indizes breiten sich aus und werden immer wichtiger. Damit wird auch die Prüfung ihrer Qualität eine immer wichtigere Aufgabe für die Politikwissenschaft. Umgekehrt bedeutet Prüfung nicht sofort Verwerfung. Indizes sind von einem immensen Nutzen für die oft auf wenige Untersuchungsfälle ausgerichtete Vergleichende Politikwissenschaft (small n problem), stellen sie doch Möglichkeiten der Informationskomprimierung bereit, ohne die weiterführende kausale Analysen manchmal gar nicht möglich wären (s. Ewert; Muno). Und so bedeutet bereits die Konstruktion eines Index einen 
Schritt auf dem Weg zu einem gesteigerten Erkenntnisgewinn, wird doch die für Wissenschaft notwendige Aufgabe der Informationsverdichtung erreicht.

Erkenntnisgewinn ist ein Prozess. Und dies gilt auch für die Verwendung von Indizes in der Vergleichenden Politikwissenschaft. Dieser Prozess ist noch nicht am Ende angelangt, sondern befindet sich auf dem Weg. Pauschale Forderungen, auf Indizes zu verzichten, sind dabei genauso wenig hilfreich, wie Forderungen, jegliche Form statistischer sozialwissenschaftlicher Analyse aufzugeben. Sie werden in der Zukunft in komplexen Gesellschaften immer mehr zur Informationsverdichtung benötigt werden, und ihre Qualität wird sich verbessern. Eine hinreichende Reflexion dessen, was man mit Indizes tut, wie man es tut und was die (politischen) Konsequenzen sein können, ist deswegen nicht weniger angebracht. Wir hoffen mit dem vorliegenden Band hierzu einen Beitrag zu leisten.

\section{Literatur}

Aarebrod, Frank H., und Pal H. Bakka. 1997. Die vergleichende Methode in der Politikwissenschaft. In Vergleichende Politikwissenschaft, Hrsg. Dirk Berg-Schlosser, und Ferdinand Müller-Rommel, 57-76. Opladen: Westdeutscher.

Castles, Francis G. 1998. Comparative public policy: patterns of post-war transformation. Cheltenham: Edward-Elgar.

Cheibub, José Antonio, et al. 2009. Democracy and dictatorship revisited. Public Choice 143: 67-101.

Fuchs, Dieter, und Edeltraud Roller. 2008. Die Konzeptualisierung der Qualität von Demokratie. Eine kritische Diskussion aktueller Ansätze. In Bedrohungen der Demokratie, Hrsg. André Brodocz, Markus Llanque, und Gary Schaal, 77-96. Wiesbaden: VS Verlag für Sozialwissenschaften.

Geddes, Barbara. 1999. What do we know about democratization after twenty years? Annual Review of Political Science 2:115-144.

Geddes, Barbara, et al. 2011. Authoritarian regimes: a new data set. Manuscript. http://dictators. la.psu.edu/data/GlobalRegimesCodebook.pdf. Zugegriffen: 1. Aug. 2012.

Hadenius, Axel, und Jan Teorell. 2006. Authoritarian regimes: stability, change, and pathways to democracy 1972-2003. Kellogg Institute Working Paper Series 331: University of Notre Dame.

Hadenius, Axel, und Jan Teorell. 2007. Pathways from authoritarism. Journal of Democracy 18: $143-156$.

Kailitz, Steffen. 2009a. Stand und Perspektiven der Autokratieforschung. Zeitschrift für Politikwissenschaft 3:437-488.

Kailitz, Steffen. 2009b. Stand und Perspektiven der Autokratieforschung. Zeitschrift für Politikwissenschaft 19:437-488.

Kailitz, Steffen. 2012. Why and how we should classify political regimes by their legitimation. Democratization forthcoming.

Lauth, Hans-Joachim. 2004. Demokratie und Demokratiemessung. Eine konzeptionelle Grundlegung für den interkulturellen Vergleich. Wiesbaden: VS Verlag für Sozialwissenschaften.

Lauth, Hans-Joachim, Gert Pickel, und Susanne Pickel. 2009. Methoden der Vergleichenden Politikwissenschaft. Wiesbaden: VS Verlag für Sozialwissenschaften.

Müller, Thomas, und Susanne Pickel. 2007. Wie lässt sich Demokratie am besten messen? Zur Konzeptqualität von Demokratie-Indizes. Politische Vierteljahresschrift 3:511-539. 
Munck, Gerardo. 2009. Measuring democracy: a bridge between scholarship and politics. New York: Johns Hopkins University.

Munck, Gerardo L., und Jay Verkuilen. 2002. Conceptualizing and Measuring Democracy. Evaluating Alternative Indices. Comparative Political Studies 35 (1): 5-34.

Nohlen, Dieter. 1994. Vergleichende Methode. In Politikwissenschaftliche Methoden, Hrsg. Jürgen Kriz, und ders., 507-517. München: Beck.

Pickel, Susanne, und Thomas Müller. 2006. Systemvermessung - Schwächen der Konzepte und Verzerrungen der empirisch-quantitativen Bestimmung von Demokratie und Autokratie. In Demokratisierung im internationalen Vergleich. Neue Erkenntnisse und Perspektiven, Hrsg. Gert Pickel, und Susanne Pickel, 135-172. Wiesbaden: VS Verlag für Sozialwissenschaften.

Pickel, Susanne, und Gert Pickel. 2006. Politische Kultur- und Demokratieforschung. Wiesbaden: VS Verlag für Sozialwissenschaften.

Przeworski, Adam et al 2000. Democracy and development: political institutions and well-being in the world, 1950-1990. Cambridge: Cambridge University Press.

Schnell, Rainer, Paul B. Hill, und Elke Esser. 2005. Methoden der empirischen Sozialforschung. München: Oldenbourg.

Teorell, Jan. 2010. Determinants of democratization. Explaining regime change in the world, 19722006. Cambridge: Cambridge University Press. 